\section{Thoracic actinomycosis: a rare occurrence}

\author{
Waqas Jehangir, ${ }^{1}$ Bhumesh Vaidya, ${ }^{2}$ \\ Souad Enakuaa, ${ }^{1}$ Nazar Raoof, ${ }^{1}$ \\ John R. Middleton, ${ }^{1}$ Abdalla Yousif ${ }^{1}$ \\ ${ }^{1}$ Raritan Bay Medical Center, Perth \\ Amboy, NJ; ${ }^{2}$ Ross University School of \\ Medicine, Miramar, FL, USA
}

\begin{abstract}
Actinomyces israelii is a branching anaerobic bacilli microorganism that can be identified as normal flora throughout various portions of the human alimentary canal. It is crucial to establish a diagnosis as treatment will vary depending on the clinical form of the disease. We report a case of a 78-year-old man who initially presented with an acute onset of respiratory distress displayed contrast leakage on computed tomography from the site of a previously inserted esophageal stent for an unsuccessful surgical repair of an esophageal rupture. In addition to the contrast leakage, the presence of a bronchopulmonary fistula imaging prompted the need for further investigation. Our patient was empirically treated with antibiotics and obtained blood cultures, which returned positive $A$. israelii.
\end{abstract}

\section{Introduction}

Among the many Actinomyces species that exist, the one most commonly responsible for human disease is Actinomyces israelii (A. israelii). ${ }^{1}$ These thin, branching, facultative anaerobic, Gram-positive bacilli are known to cause various clinical forms of actinomycosis. These include the oral-cervicofacial form (most common), which is characterized by a painless lumpy jaw, the thoracic form, presents as a mass that extends to the chest wall and is often misdiagnosed as a primary malignancy, the pelvic form (usually presents in women), and the abdominal form. ${ }^{1,2}$ A. israelii is an opportunistic pathogen that colonizes the human mouth, digestive tract, and vagina. ${ }^{3}$ It establishes infection through a breach in the integrity of the mucosal membrane during dental or gastrointestinal tract procedures, from aspiration, or digestive tract diseases. ${ }^{1,2}$ As the disease progresses, the abscesses can enlarge and penetrate surrounding tissues causing purulent leakage. ${ }^{2}$ Since the presentation and treatment can vary depending on the form of actinomycosis, it is important to consider A. israelii in the differential diagnosis; especially if the patient's medical history includes any of the aforementioned risk factors. We describe a case of the thoracic form of actinomycosis that was correctly diagnosed after performing detailed clinical and microbiological studies.

\section{Case Report}

A 78-year-old Caucasian man who is statuspost esophageal rupture, was transported by ambulance from a long term care facility for a gastroenterology appointment. En route to the facility, he had sudden onset respiratory distress accompanied chest and back pain. The patient became hypotensive and tachycardic and was evaluated in the emergency department. His past medical history includes COPD, atrial fibrillation, Clostridium difficile infection, and empyema with mediastinitis. He has a past surgical history of esophageal repair and gastrostomy tube insertion. The patient was born in the U.S and has no history of recent travel. On physical examination, the patient's vital signs were as follows: blood pressure $100 / 60 \mathrm{mmHg}$, hear rate $80 / \mathrm{min}$, respirations $16 / \mathrm{min}$, temperature $99^{\circ} \mathrm{F}$. He had decreased bilateral air entry upon lung auscultation with dullness to percussion of the right lung. The patient was admitted to the intensive care unit with the diagnosis of acute respiratory distress secondary to acute diastolic decompensation. He was started on 50\% oxygen via mask, ordered to have nothing by mouth, and placed on enoxpaparin, amiodarone, and digoxin. However, the patient subsequently developed respiratory failure and was intubated. His complete blood count showed a mild leukocytosis of $13.8 \mathrm{~K} / \mathrm{uL}$ with $85 \%$ neutrophils, hemoglobin $9.1 \mathrm{~g} / \mathrm{dL}$, hematocrit $27.6 \%$ and platelet $468.0 \mathrm{~K} / \mathrm{uL}$. Serum chemistries were within normal limits. Urine cultures, sputum cultures, and three sets of blood cultures were obtained. He was treated empirically for septic shock with intravenous vancomycin, cefepime, and clindamycin. Computed tomography demonstrated persistent esophageal leakage of contrast from the lower portion of his esophageal stent with filling of the right lobe bronchus, consistent with a bronchopulmonary fistula (Figure 1). Of the three sets of blood cultures obtained, one was positive for A. israelii.

\section{Discussion}

Actinomycosis can be found worldwide, especially in areas that have suboptimal standards of dental care. ${ }^{4}$ Although the pathogenesis is not completely understood, previously
Correspondence: Waqas Jehangir, Raritan Bay Medical Center, 530 New Brunswick Ave, Perth Amboy, NJ 08861, USA.

Tel.: +1.267.8447119

E-mail: wjehangir@hotmail.com

Key words: Thoracic actinomycosis; A. israelii; bronchopulmonary fistula.

Contributions: the authors contributed equally.

Conflict of interest: the authors declare no potential conflict of interest.

Received for publication: 21 April 2015.

Revision received: 9 February 2016.

Accepted for publication: 12 February 2016.

This work is licensed under a Creative Commons Attribution-NonCommercial 4.0 International License (CC BY-NC 4.0).

(C) Copyright W. Jehangir et al., 2016

Licensee PAGEPress, Italy

Infectious Disease Reports 2016; 8:5963

doi:10.4081/idr.2016.5963

reported cases of patients with positive $A$. israelii blood cultures typically have a documented past medical history revealing either an appendectomy, perforation of an abdominal organ, past surgery, or intrauterine device use. ${ }^{4}$ In our case, it is presumed that the patient's esophageal stent placement site was the nidus of the $A$. israelii infection that complicated further to involve the lungs, which eventually resulted in the formation of a bronchopulmonary fistula. Our patient's previous history of an unsuccessful esophageal surgical repair and stent placement supports the hypothesis that this portion of the gastrointestinal tract is the most likely source of his $A$. israelii bacteremia. Time for symptoms to appear may range from one month to two years due to the indolent nature of disease. ${ }^{4}$ The associated findings can be non-specific, which include pain, weight loss, anorexia, fever, chills, constipation, leukocytosis, palpable mass, or fistulas. ${ }^{4}$ The presentation of actinomycosis can mimic certain chronic diseases such as tuberculosis, carcinoma, sarcoma, or inflammatory bowel disease. ${ }^{4}$ Although determining the source of bacteremia can be difficult, microscopic analysis of tissue infected with A. israelii may reveal suppurative and granulomatous inflammation, proliferation of connective tissue, or presence of dense coaggregations known as sulfur granules. ${ }^{1}$ Identification of sulfur granules are present in approximately half the cases of actinomycosis and are not considered pathognomonic since other microorganisms, such as Nocardia and Streptomyces can aggregate and resemble sul- 
fur granules found in this infection. ${ }^{4}$ Drug resistance is currently not a point at issue for medical management of the Actinomyces spp., which has demonstrated maximal susceptibility to beta-lactams, especially amoxicillin or penicillin G. ${ }^{5}$ Therefore, amoxicillin or penicillin $\mathrm{G}$ are antibiotics of choice in the treatment of actinomycosis. ${ }^{5}$ High doses of the antibiotics over a prolonged time frame is an important underlying principle that should be taken into consideration when treating the disease. ${ }^{6}$ Therapy should be tailored to the individual, but a recommended guideline for serious infections involves using 18 to 24 million units of penicillin IV for 2 to 6 weeks, followed by oral therapy with penicillin or amoxicillin for 6 to 12 months. ${ }^{6}$ Since Actinomyces spp. are do not produce beta-lactamases, it serves no purpose to combine amoxicillin with clavulanic acid unless other co-existing pathogens are involved. ${ }^{5}$ Thus, it would be essential to consider the site of infection when constructing empirical therapy. A previous study reports experience in treating all forms of actinomycosis with shorter courses of therapy. ${ }^{7}$ However, it's recommended particularly in patients with bulky thoracic and/or abdominal actinomycosis, should not receive a shortcourse therapy unless surgical debulking is first performed. ${ }^{7}$ Kinnear and MacFarlane described 19 patients in whom thoracic actinomycosis was cured with a median duration of antibiotic therapy of 6 weeks (range, 1 week to

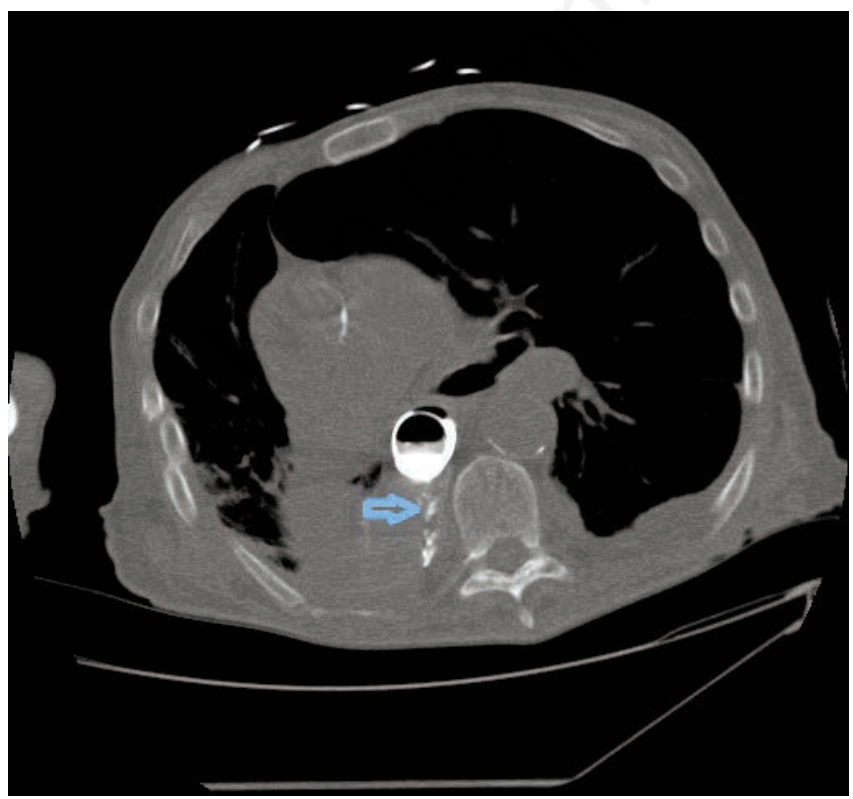

Figure 1. Computed tomography demonstrating persistent esophageal leakage of contrast from the lower portion of his esophageal stent with filling of the right lobe bronchus, consistent with a bronchopulmonary fistula.
6 months). Surgical resection was performed for 7 patients. ${ }^{7}$ Hsieh et al. successfully treated thoracic actinomycosis in 16 patients with 2 weeks of intravenous penicillin therapy, followed by 3 months of oral penicillin therapy. ${ }^{7}$ Nine of the patients underwent surgical debulking. ${ }^{7}$ Relapse is known to be one of the clinical hallmarks of actinomycosis. ${ }^{6}$ Extending treatment past the resolution of measurable disease will minimize the likelihood of relapse. ${ }^{6}$ Alternative treatment with tetracycline in penicillin-allergic patients has largely been used with the most success and for allergic pregnant patients, erythromycin is a suitable alternative. ${ }^{6}$ Although there is minimal clinical evidence on the newer antimicrobial agents, anecdotal success has been reported with the use of piperacillin-tazobactam, imepenem, or ceftriaxone.,5,8,9 Due to their lack of efficacy suggested by in vitro data, metronidazole, aminoglycosides, oxacillin, dicloxacillin, and cephalexin should be avoided in treating actinomycosis.,

\section{Conclusions}

Although rare, it is imperative for physicians to consider thoracic actinomycosis in their differential diagnosis. ${ }^{4}$ A previous study that presents a retrospective case series of 28 patients with histologically confirmed thoracic actinomycosis evaluated the optimal duration of IV and oral antibiotic treatment concluded that thoracic actinomycosis is best treated with individualized therapeutic modalities. ${ }^{10}$ These therapeutic modalities depended on factors such as the initial burden of the disease, performance of resectional surgery, and the clinical and radiological responses to therapy. ${ }^{10}$ For patients with confirmed thoracic actinomycosis, the initial treatment should begin with the recommended guideline followed by tailoring the therapy based on the clinical assessment of an individual's response to therapy.

\section{References}

1. Alekh K, Dazley J, Sison R, Slim J, Boghossian J. A rare case of Actinomyces israelii bacteraemia. JMM Case Reports 2014;1:000158.

2. Fahim A, Teoh R, Kastelik J, et al. Case series of thoracic actinomycosis presenting as a diagnostic challenge. Respiratory Med CME 2009;2:47-50.

3. Smith A, Hall V, Thakker B, Gemmell C. Antimicrobial susceptibility testing of Actinomyces species with 12 antimicrobial agents. J Antimicrob Chemother 2005;56: 407-9.

4. Coban A, Yetkin G, Kebudi A. Abdominal actinomycosis: a case report. Acta Chir Belg 2003;103:521-3.

5. Ferry T, Valour F, Karsenty J, et al. Actinomycosis: etiology, clinical features, diagnosis, treatment, and management. Infect Drug Resist 2014;7:183-97.

6. Bennett J, Dolin R, Blaser M. Mandell, Douglas, and Bennett's principles and practice of infectious diseases. 8th ed. Philadelphia, PA: Elsevier; 2015.

7. Sudhakar S, Ross J. Short-term treatment of actinomycosis: two cases and a review. Clin Infect Dis 2004;38:444-7.

8. Yew WW, Wong PC, Lee J, et al. Report of eight cases of pulmonary actinomycosis and their treatment with imipenem-cilastatin. Monaldi Arch Chest Dis 1999;54:1269.

9. Onal ED, Altinbas A, Onal IK, et al. Successful outpatient management of pelvic actinomycosis by ceftriaxone: a report of three cases. Braz J Infect Dis 2009;13:391-3.

10. Choi J, Koh WJ, Kim TS, et al. Optimal duration of IV and oral antibiotics in the treatment of thoracic actinomycosis. Chest 2005;128: 2211-7. 